\section{Allergologisches Nachschlagewerk für die Praxis}

\begin{abstract}
- lf Jahre nach Erscheinen der Erstauf-
Clage liegt die „Praktische Allergologie" nun in gründlich überarbeiteter Form vor. Zwischenzeitlich haben allergische Erkrankungen quantitativ einen noch höheren Stellenwert erhalten, das Spektrum diagnostischer und therapeutischer Möglichkeiten hat sich ebenfalls erweitert. Aufgrund der Vielzahl von Manifestationsformen der Allergie sind sehr unterschiedliche medizinische Fachbereiche involviert, dabei den interdisziplinären Überblick zu behalten ist für den „Praktiker“ kaum
\end{abstract}

umsetzbar. Hier setzt das vorliegende Buch sehr praxisnah an. Die einzelnen Beiträge dieses Multiautorenwerks decken das gesamte Spektrum möglicher Symptome und Krankheitsbilder vom Abdominalschmerz beim Latex-Nahrungsmittel-Allergiesyndrom bis zur Zimmerspringbrunnenalveolitis ab. Neben Genetik und Umwelteinflüssen werden die immunologischen Grundlagen, die allergischen Krankheitsbilder nach Fachgebieten bzw. Organen geordnet, Diagnostik und Therapie kurz und gut verständlich dargestellt. Jedes

\title{
Ratgeber für ein leidiges Problem
}

$\mathrm{D}_{\mathrm{u}}^{\mathrm{ie}}$ ie Herausgeberin ist Juristin und Unternehmensberaterin und spezialisiert auf das „Forderungsmanagement Selbstständiger". Den meisten von uns ist die Zahlungsverweigerung von Patienten mehr oder weniger bekannt, das Handling stellt viele jedoch vor Probleme, die anderen Berufsgruppen fremd sind. Im Vorwort wird dieser Tatbestand sehr treffend als „Gratwanderung zwischen standes- und berufsrechtlichen Vorschriften einerseits und effektiven außergerichtlichen wie gerichtlichen Maßnahmen andererseits" beschrieben.
Das Buch beginnt treffenderweise mit einer Darstellung des Wandels vom Arzt zum Unternehmer, ein Paradigmenwechsel, der vielfach noch nicht realisiert worden ist, dessen Bewusstmachung für das wirtschaftliche Überleben heute aber entscheidend sein kann. Weitere Kapitel befassen sich mit den Themen ärztliche Rechts- und Standespflichten, erfolgreiches Forderungsmanagement sowie der gerichtlichen Geltendmachung als letzter Möglichkeit, erbrachte Leistungen einzutreiben.

Sehr wichtig für den praktischen Umgang sind schließlich auch die abschließenden Beiträge zu den "Schuldnertricks“
Hauptkapitel wird durch ein übersichtliches und weitgehend aktuelles Literaturverzeichnis ergänzt. Als aktuelles Nachschlagewerk kann das Buch dem allergologisch tätigen Arzt vorbehaltslos empfohlen werden.

gg

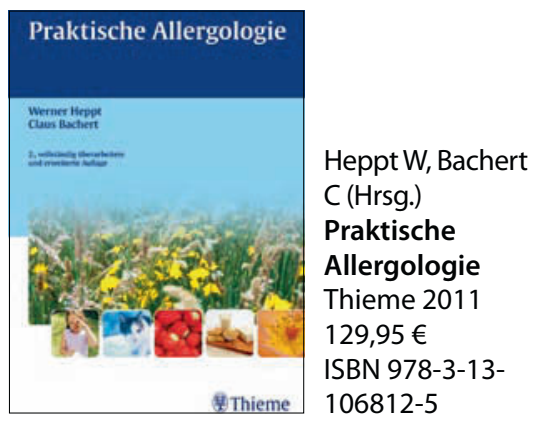

(Stichwort u. a: „wir haben nicht über den Preis gesprochen“ oder „unbekannt verzogen“) und dem Stellenwert des Outsourcing der Forderungsbetreibung über Verrechnungsstellen oder Inkassounternehmen.

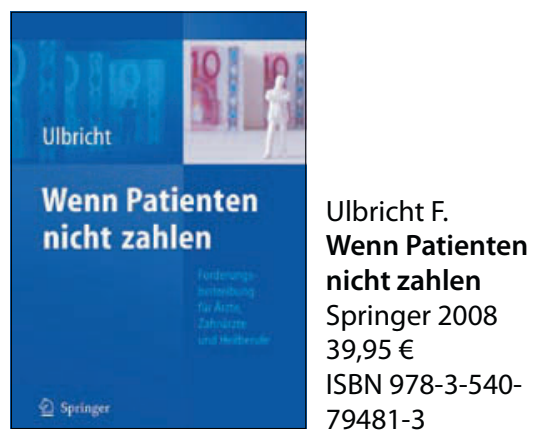

\section{Einsatzmöglichkeiten der Neuraltherapie}

$\mathrm{D}$ as Buch gibt dem Leser aus unterschiedlichen Perspektiven umfassende Einblicke in die Einsatzmöglichkeiten der Neuraltherapie. Beginnend mit einem Überblick über die historische Entwicklung und den Stellenwert der Neuraltherapie in der Geschichte der Medizin vermitteln die folgenden Kapitel detailliert die für das Verständnis der Neuraltherapie wesentlichen Grundlagen aus Anatomie, Physiologie und Pharmakologie.

Nach einem weiteren, sehr anschaulichen Kapitel über die diagnostischen Verfahren wird der Leser im Kapitel „Injektionstechniken“ durch die neuralthera- peutisch zugängliche Anatomie ,geleitet" und zwar unter Angabe von Indikationen, Kontraindikationen und spezieller Wirkungsweise.

"Neuraltherapie in den Fachgebieten" stellt dann noch einmal den unmittelbaren Bezug zu den einzelnen Fachgebieten her und listet die jeweils möglichen und vor allem sinnvollen Indikationen auf. Abschließend findet der Leser die Indikationen alphabetisch geordnet, unter Angabe von Behandlungsplanung und begleitenden Therapieoptionen. Für die Anwendung des Buchs in der Praxis ergibt sich daraus die Problematik, dass sich der Leser die benötigten Informationen zu einem Krankheits- bild immer aus unterschiedlichen Kapiteln zusammensuchen muss. Insgesamt ist das Buch als Nachschlagewerk vor allem für die Kolleginnen und Kollegen unverzichtbar, die ihren Patienten die Neuraltherapie als Therapieoption anbieten möchten.

gg

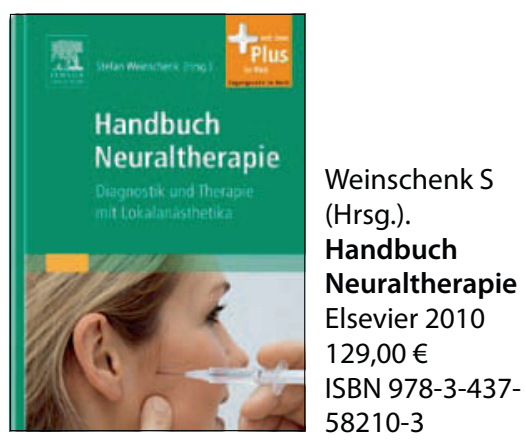

